Supporting Information:

\title{
Versatile Miniature Tunable Liquid Lenses Using Transparent Graphene Electrodes
}

\author{
Ali Shahini, ${ }^{*} \dagger$ Jinjun Xia,${ }^{\dagger}$ Zhixian Zhou, ${ }^{\dagger}$ Yang Zhao, ${ }^{\dagger}$ Mark Ming-Cheng Cheng ${ }^{*}{ }^{\dagger}$ \\ 'Department of Electrical and Computer Engineering and "Department of Physics and Astronomy, Wayne State University, Detroit, \\ Michigan 48202, United States.
}

*E-mail: ali.shahini@wayne.edu, mcheng@wayne.edu

\section{COMSOL SIMULATIONS}

This model simulates the dynamic behavior of the two phases until it reaches the equilibrium. In order to study the liquid dynamic movement driven by electrowetting effect, we did a COMSOL simulation with a voltage switched from 0 to $100 \mathrm{~V}$. In this simulation, the physics model is based on Navier-Stokes equations with a constraint of electrowetting final contact angle obtained from equation 1 in the main text. The Navier-Stokes equations govern the motion of the two-phase fluids (air and liquid) under several different forces including liquids own inertial forces, pressure forces, viscous forces and body forces. The equation 1 (in the main text) can be used to modify the final contact angle dynamically by applying the voltage and with appropriate boundary conditions. In other words, the electrowetting effect is given as a boundary condition to this model when the system is in equilibrium. The final equilibrium shape of the droplet is reached after all these forces are balanced during time. The time intervals for the simulation is $1 \mathrm{~ms}$. This model helps to see how do different liquids with different densities and different viscosities reach the final contact angle. The boundary conditions that are used for simulation consist of: 
Wall-fluid interface (the blue dots in figure S1) where the final contact angle is defined from equation 1 (in the main text) after switching the voltage.

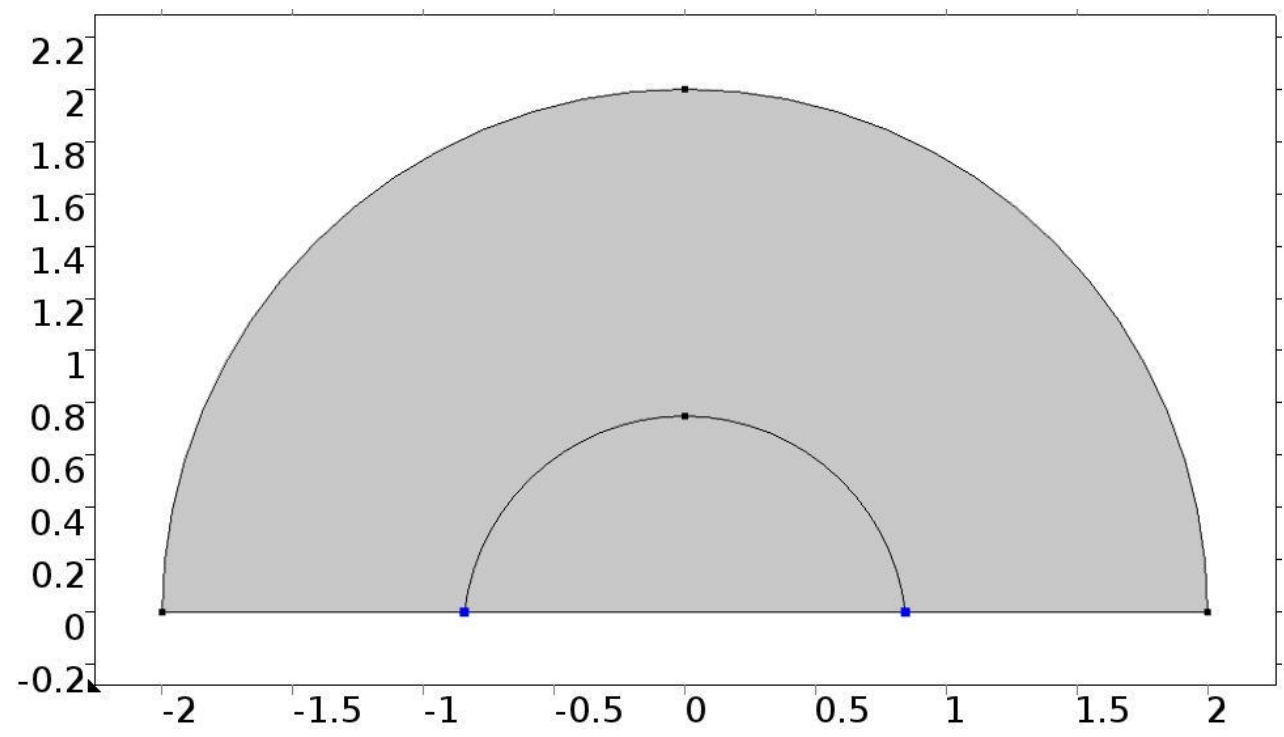

Figure S1. Wall-Fluid interface

Prescribed mesh deformation (the blue arc in figure S2) where the mesh is fixed.

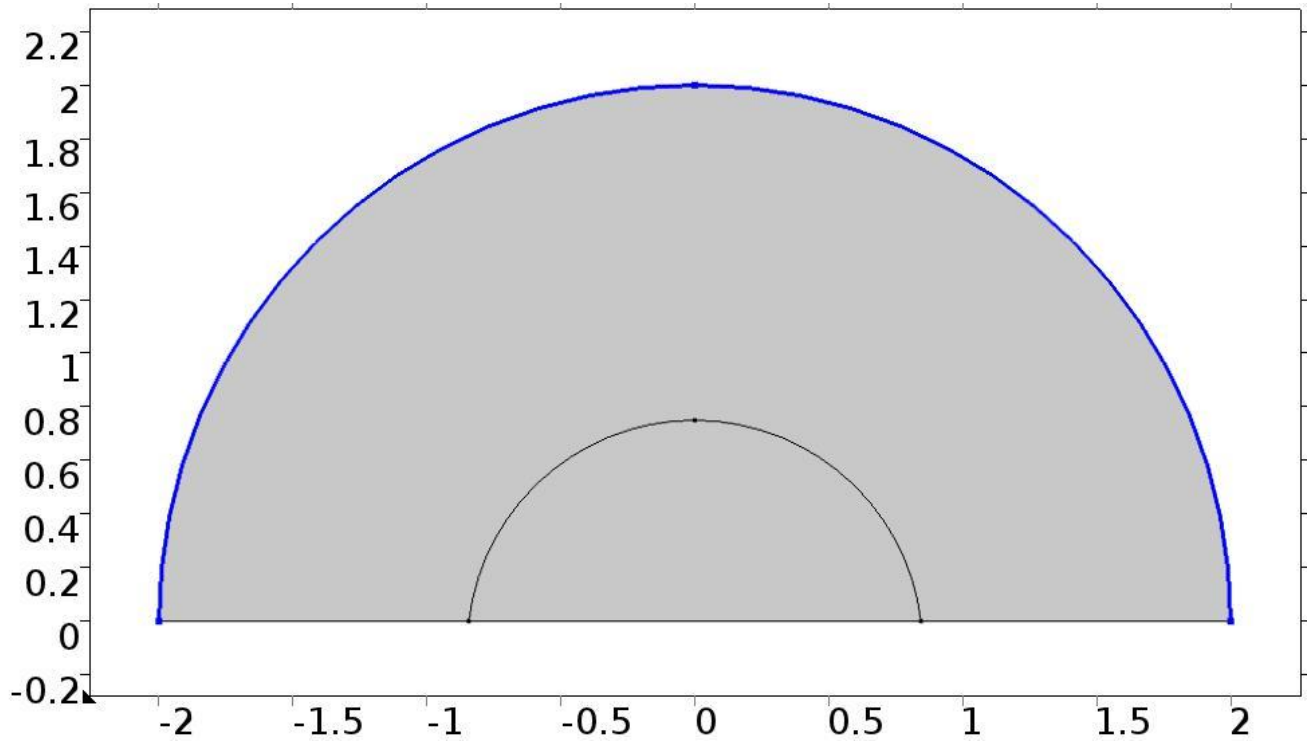

Figure S2. Prescribed mesh deformation 
Navier Slip boundary condition (The blue line in figure S3) in which the contact point is allowed to move.

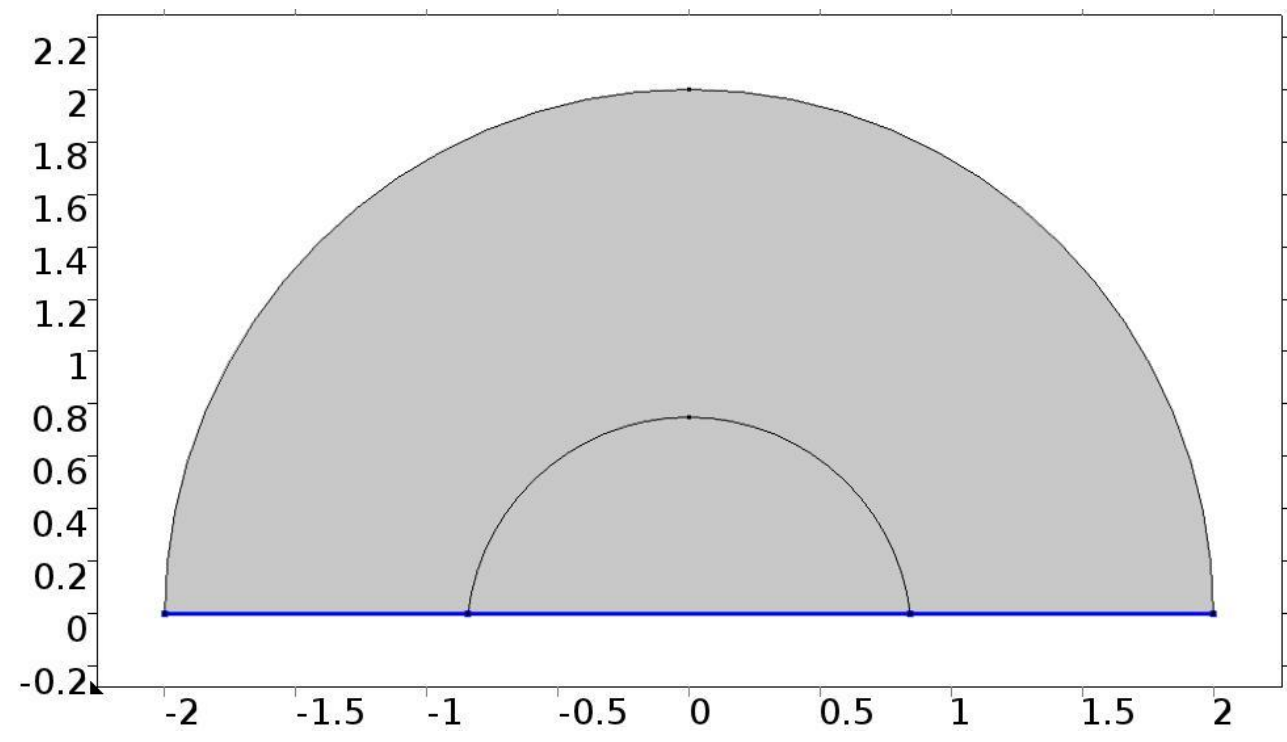

Figure S3. Navier Slip boundary condition

Fluid-fluid interface (The blue arc in figure S4) in which we define the surface tension and the equal fluid velocity for both fluid at this boundary.

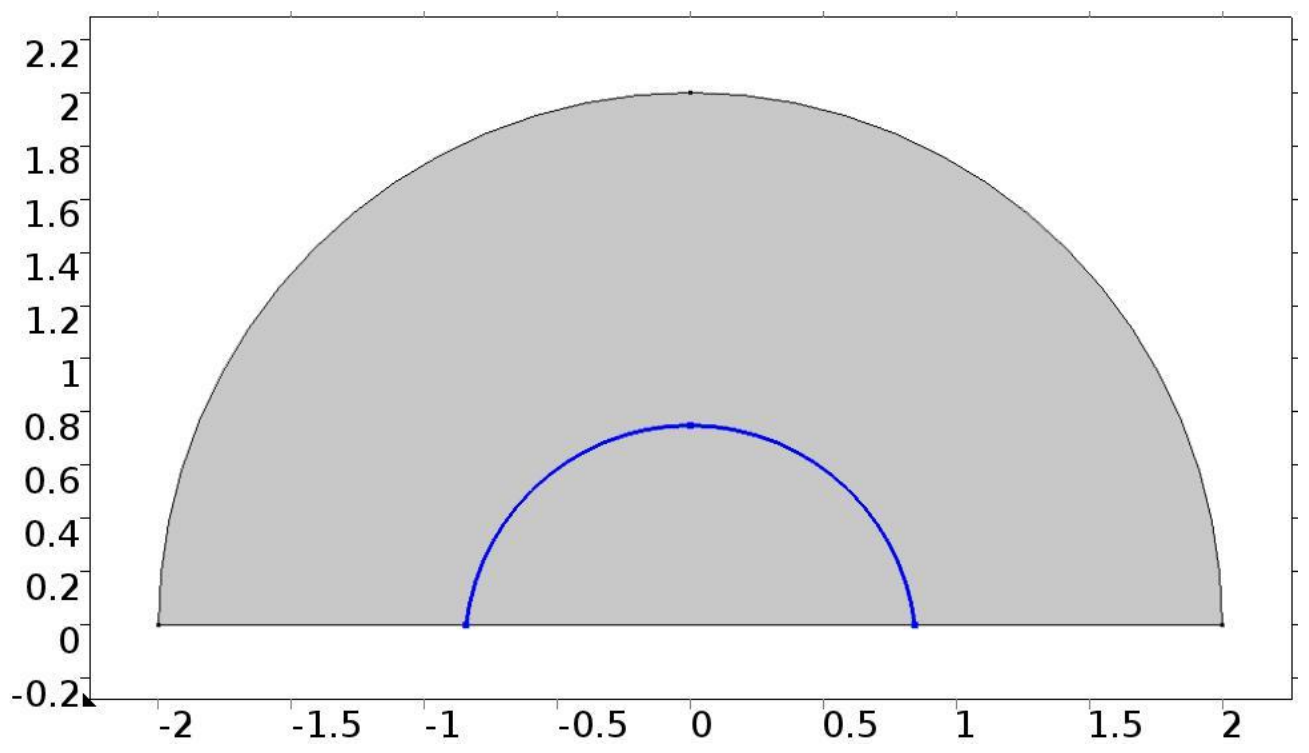

Figure S4. Fluid-Fluid interface 
Simulation of dynamic behavior of $10 \mathrm{mM} \mathrm{KCl}$ solution in the air shows that the droplet oscillates around the final position when the voltage is switched from 0 to $100 \mathrm{~V}$.
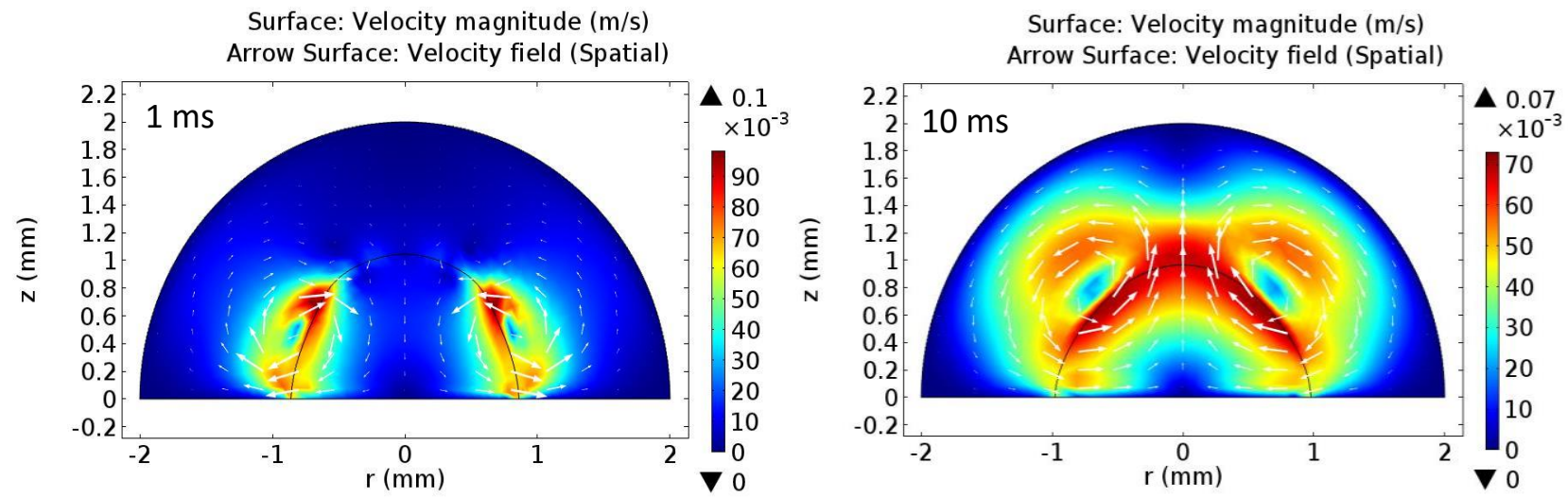

Surface: Velocity magnitude $(\mathrm{m} / \mathrm{s})$

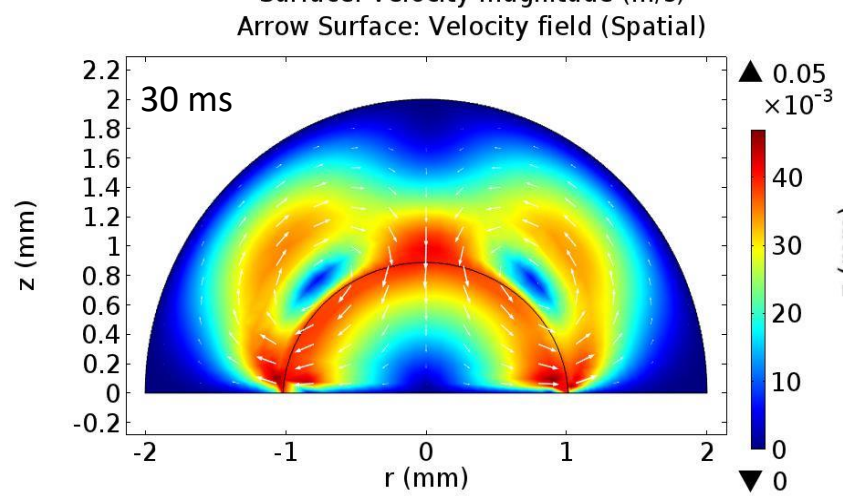

Surface: Velocity magnitude $(\mathrm{m} / \mathrm{s})$ Arrow Surface: Velocity field (Spatial)

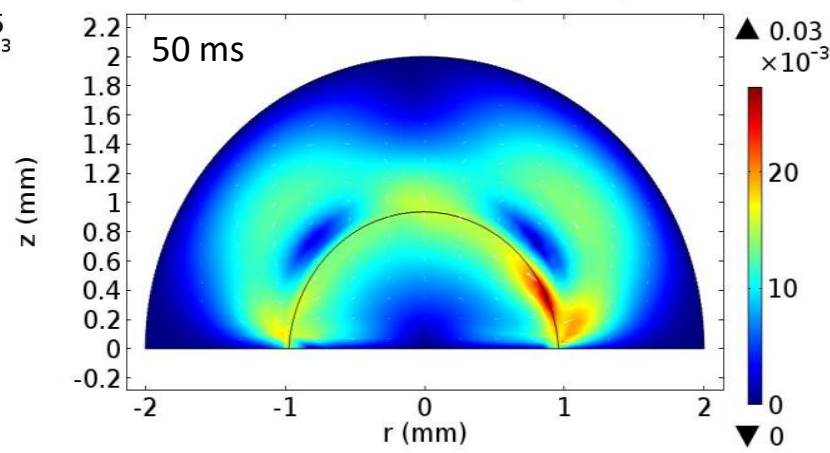

Figure S5. COMSOL simulation for $\mathrm{KCl}$ solution in the air a few milliseconds after voltage is switched from 0 to $100 \mathrm{~V}$.

The COMSOL simulation demonstrates the droplet behavior towards the final position. Note that, the contact angles in figure. $2 \mathrm{~b}$ in the main text are not defined by equation 1 but are simulated. The final contact angle which occurs after almost $150 \mathrm{~ms}$ for ionic liquid is defined by equation 1 at equilibrium. The simulation starts with initial values and boundary conditions to calculate the pressure and the velocity during time. The velocity and the pressure are in the direction that forces the contact points towards the final contact angle. Different fluids respond in different way. Figure S6 shows the ionic liquid response without any oscillation. 


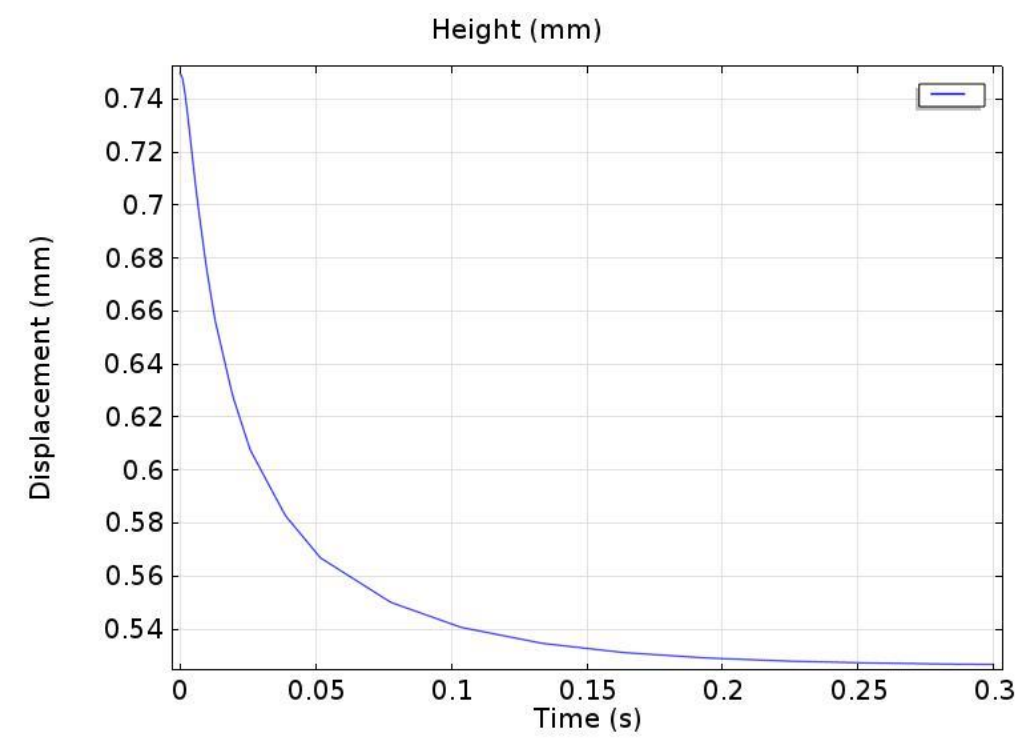

Figure S6. COMSOL simulation of ionic liquid response time to an applied voltage of $100 \mathrm{~V}$.

The $\mathrm{KCl}$ droplet responded faster to applied voltage compared to ionic liquid but oscillated before reaching the final contact angle.

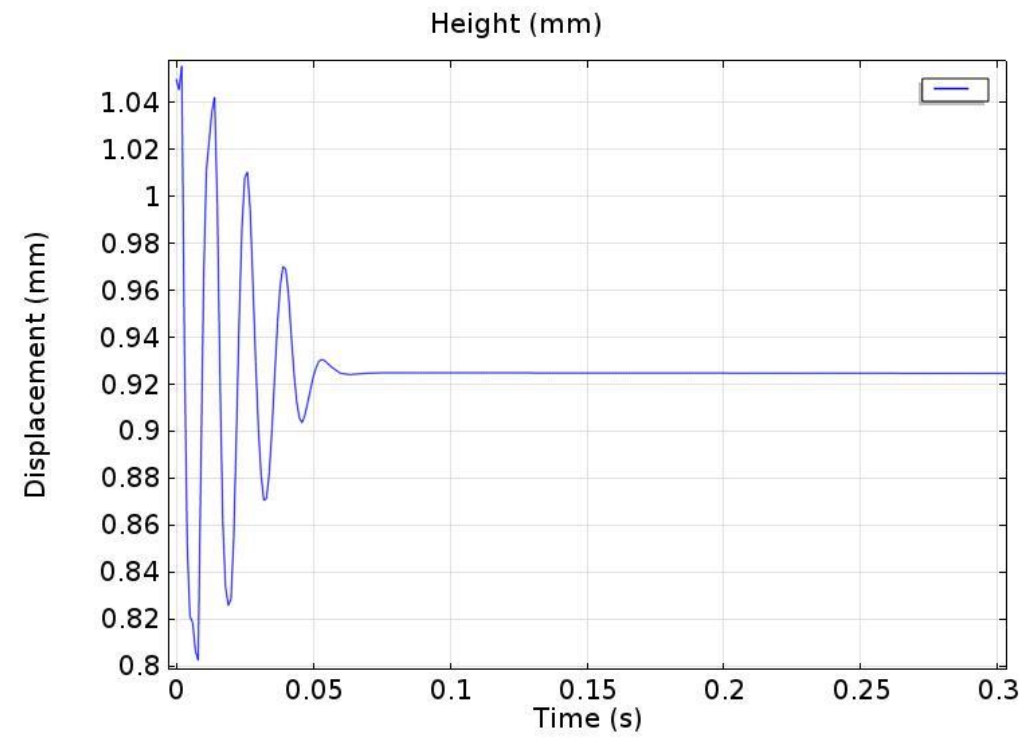

Figure S7. COMSOL simulation of $\mathrm{KCl}$ droplet response time to an applied voltage of $100 \mathrm{~V}$. 


\section{Raman spectra}

Raman spectra of CVD grown graphene with 2 peaks at $\sim 1580 \mathrm{~cm}^{-1}$ (G band) and $\sim 2680 \mathrm{~cm}^{-1}$ (2D band) with a ratio of $\frac{I_{G}}{I_{2 D}} \approx \mathbf{0 . 4}$ indicates the grown graphene is single layer.

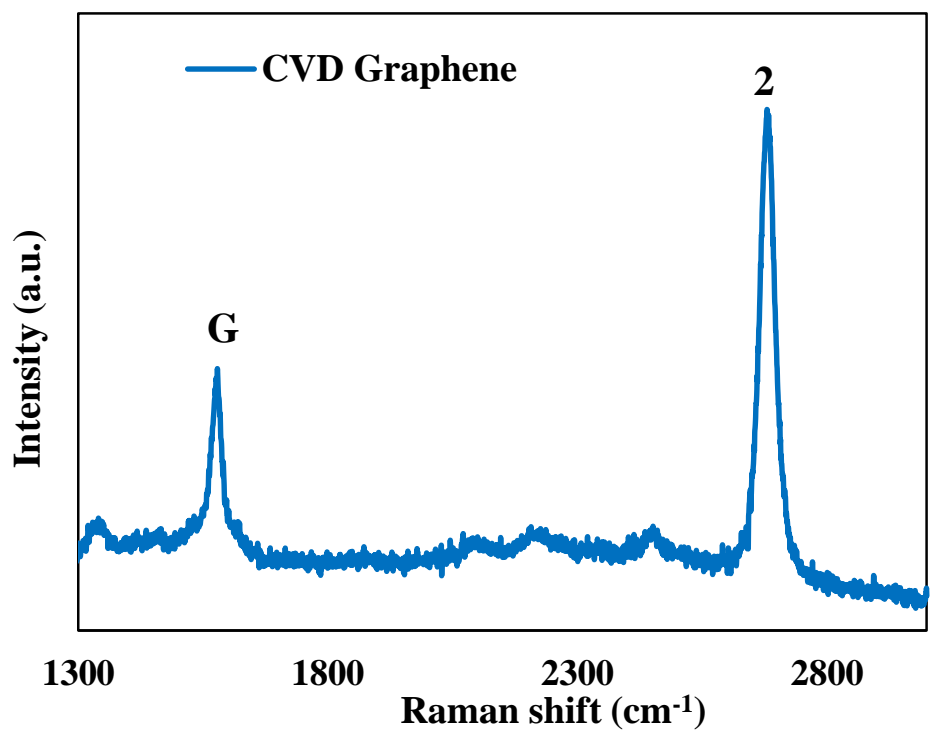

Figure S8. Raman spectra of CVD grown graphene 